\title{
Un recorrido por la educación actual. Cambios necesarios
}

\section{A Journey Through Education Today. Changes Required}

\author{
Zuleyka Suárez Valdés-Ayala ${ }^{1}$ \\ Escuela de Matemática \\ Instituto Tecnológico de Costa Rica \\ Cartago, Costa Rica \\ zsuarez@itcr.ac.cr
}

Recibido 03 de junio de $2011 \bullet$ Aceptado 26 de agosto de 2011

Resumen. Este ensayo pretende sensibilizarnos acerca de los cambios que necesita la educación en la búsqueda constante de calidad y formación de seres integrales. Para ello se mencionan ocho aspectos fundamentales que requieren su aplicación en un mediano o corto plazo, si realmente se quieren avances significativos, sin olvidar que el cambio debe ser permanente.

Palabras claves. Educación, cambios, mejoras, igualdad, futuro.

Abstract. This essay aims at raising awareness on the changes required in Education to continuously search for quality improvement and the formation of integral human beings. Eight fundamental aspects are included in this paper. Such aspects need to be applied in the short- or medium term to have a significant progress, keeping in mind that the change must be permanent.

Keywords. Education, changes, improvements, equality, future.

\section{Introducción}

En el mundo entero y a través de los años se han venido haciendo esfuerzos para generar cambios en la educación. Estos esfuerzos, aunque son de aplaudir, no han sido suficientes y se siguen presentando enormes brechas y deficiencias.

\footnotetext{
Máster en educación con énfasis en docencia universitaria (UNA) y Licenciada en la Enseñanza de la Matemática (UNA). Laboró durante 4 años en la enseñanza matemática a nivel de primaria y durante 7 años en la enseñanza matemática a nivel de secundaria y educación diversificada. Desde hace 4 años es docente e investigadora en la Escuela de Matemática del ITCR.
} 
Al respecto De Zubiría (s. f.) afirma que: “(...) la escuela tradicional se torna obsoleta frente a los dramáticos cambios sociales, económicos y políticos vividos desde hace más de cuatro décadas. Se torna ineficiente e inadecuada. No porque siempre lo haya sido, sino porque la sociedad cambiante le plantea nuevos retos y nuevas demandas." (p. 1)

Por lo anterior se requiere de una educación de calidad, la cual tiene que ver con que los jóvenes adquieran ciertas competencias claves, entre ellas: competencia para la vida, la cual implica, según Hoyos, Delgado y Peré (s. f.), la renovación completa de los métodos de enseñanza.

Entonces, se hace necesario apostar por nuevos cambios que generen mayores transformaciones, que busquen la formación de un ser integral que, como menciona Naranjo (2004), sea una educación del cuerpo, las emociones, la mente y el espíritu para devolver al mundo seres capaces de servir con generosidad. "Lejos de constituir un lujo, una educación nueva (...) es una necesidad urgente, y es también nuestra mayor esperanza: todos nuestros problemas se simplificarían enormemente (...)" (Naranjo, 2004, p. 5).

Se sigue dando énfasis a aspectos no tan importantes, hay preguntas que aún quedan sin respuesta, incluso en este ensayo. Estas siempre siguen apareciendo en cada mesa redonda, panel, congreso e investigación, dos de ellas son: ¿Cómo se mide la calidad de la educación? ¿Son suficientes las pruebas de bachillerato utilizadas en Costa Rica que promocionan a los estudiantes, cuyos resultados no se utilizan adecuadamente?

\section{Contenidos}

\section{Apostarle a cambios inmediatos}

De nada sirve ampliar la cobertura y reformar el currículo si la calidad de la educación, la que se imparte directamente en el recinto escolar, es deficiente. Al respecto se mencionan dos citas relevantes que revelan situaciones sobre las cuales actuar en forma inmediata:

Los niveles de calidad que alcance la educación costarricense serán tan altos, como alta sea la calidad de los docentes y directores en cuyas manos descansa la trascendental tarea institucional (...). (Consejo Superior de Educación, 2008, p. 12)

(...) El aula es el espacio donde se efectúan los mayores esfuerzos por aprender y donde el sistema educativo tiene la mayor cuota de responsabilidad de hacer bien su trabajo (...). (Walter, 2000, p. 37)

Las frases hablan por sí solas, los cambios no pueden ser solo a nivel macro, se requiere de mejoras también en el personal que interactúa directamente con los estudiantes en el aula.

Para educarse hoy, se requiere además, según Rivera (2005), “(...) adaptarse cultural, social, laboral, profesional y personalmente al ritmo del cambio, [basándose en] nuevas concepciones culturales, de producción, de relaciones sociales, económicas e industriales (...)” (p. 5).

De lo anterior se deduce que también el estudiante debe poner de su parte para lograr estas mejoras, pero para ello se necesitan docentes preparados y capaces, convertidos en facilitadores del cambio y que estén dispuestos a constantes actualizaciones. 
Al respecto, Tedesco (2003) apuesta por un docente comprometido con su profesión, que no vaya "(...) de un establecimiento a otro sin tener en cuenta las características individuales de sus alumnos ni el perfil institucional” (p. 12), ya que esto atentaría contra los propósitos de formar un ser integral.

Se requieren, por tanto, docentes con una formación sólida, un compromiso real y una vocación genuina, dispuestos a seguir formándose continuamente y que se constituyan en ejemplo para sus estudiantes, mediante una relación cognitiva y afectiva con estos.

Junto al cambio en el docente como persona y en su formación, se necesita también una serie de condiciones si se quiere formar un ser integral. Algunas de ellas se enuncian a continuación y es importante resaltar que todas van estrechamente vinculadas en esta búsqueda de mayores logros educativos:

\section{Preparar al individuo no para almacenar contenidos sino para adquirir competencias}

Este es un reto importante que debemos asumir. El aprender a aprender debe ser una prioridad hoy. Tedesco (2003) ahonda al respecto y resalta la importancia del desarrollo cognitivo orientado hacia la capacidad de construir los aprendizajes, los valores y la identidad propia y, por otro lado, De Zubiría (s. f.) afirma muy acertadamente que “(...) el mundo contemporáneo exige la formación de individuos con mayor capacidad para el análisis, la interpretación y la síntesis" (p. 4). Se puede agregar que este individuo debe ser autónomo y en esa capacitación constante ir formando su propio proyecto de vida.

\section{Estar anuentes a reformas curriculares necesarias}

Como afirma Gómez (2010), la realidad no puede ser concebida como entidad final acabada, esta cambia, es un proceso y. por ende. la educación debe ajustarse a esos cambios. Ya no podemos pensar en un profesor que transmite y un alumno pasivo que como envase "recibe conocimientos hasta llenarse". La educación, necesita ir mucho más allá de la mera transmisión de información. Al respecto, De Zubiría (s. f.) asevera que más que el conocimiento, se torna como prioritaria la capacidad para comprenderlo, para interpretarlo y para procesarlo.

Este conocimiento, como dice Morin (1999), no está exento de errores e ilusiones; por lo tanto, debemos aprender a reconocerlo, a examinarlo y esto solo se logra con habilidades, aptitudes y competencias bien definidas para formar individuos flexibles y de mentalidad amplia.

Naranjo (2004) por su parte afirma que: “(...) no podemos permitirnos sobrecargar la capacidad de almacenamiento de nuestros cerebros con informaciones detalladas sobre cosas o aspectos no esenciales, sino que debemos concentrarnos al máximo en cuestiones realmente significativas (...)” (p. 10).

Se debe, entonces, apostar por un currículo basado en competencias, actualizado, significativo y pertinente, pero contextualizado de acuerdo con la región donde aspectos que no resulten significativos para el estudiante sean eliminados. 


\section{Mejorar las condiciones de vida para lograr mejoras en la educación}

En una encuesta realizada por el Reino Unido a 110 países (90\% de la población mundial) que mide la economía, la situación empresarial, la seguridad, el gobierno, la salud, la libertad personal y el capital social, los cuales son factores de la prosperidad de un país (índice de prosperidad Legatum), Costa Rica ocupa el puesto 33, lugar nada despreciable. Noruega y Dinamarca se sitúan en los dos primeros lugares (países con altas tasas de impuestos) y Canadá la séptima posición (Arias, 2010). ¿Qué hay que aprender de estos países?

La clave no es copiar un modelo y aplicarlo tal cual, pues esto en vez de ayudar, podría tornarse en un mayor fracaso, pero sí debe existir una retroalimentación para poder efectuar mejoras en nuestro sistema económico y social, lo cual obviamente repercute directamente en la educación.

Coincidiendo con Walter (2000), para pensar en mejoras educativas, se debe entender la educación como un sistema cuyas partes se interrelacionan y refuerzan o debilitan mutuamente

En Costa Rica se han hecho esfuerzos considerables en este punto. Uno de ellos es el Programa Avancemos, que otorga becas a estudiantes de bajos recursos para que no deserten del colegio y continúen sus estudios. El otro es la campaña reciente titulada "libros para todos". Está claro que sin las condiciones necesarias y los materiales adecuados, el éxito en la educación no es posible.

\section{Lograr una mayor igualdad entre países de la región}

Ya sea mediante mejoras internas a nivel de país o buscando ayuda de organismos internacionales, es necesario lograr una mayor equidad entre países de la región.

En el informe del 2010 del Programa de las Naciones Unidas para el Desarrollo (PNUD), en los últimos 40 años, ningún país de la región latinoamericana ha estado entre los 20 mejores. Chile ocupa el primer puesto entre los países latinoamericanos, y el lugar 45 a nivel mundial, Costa Rica ocupa el puesto 62, Nicaragua el puesto 106 y Guatemala (el peor calificado), el lugar 116 de un total de 169 países (PNUD, 2010).

Estas posiciones deben llevar a una reflexión en Latinoamérica y, como plantea Walter (2000), con quien se coincide, la educación que se recibe en Centroamérica es deficiente, el tiempo que permanecen los estudiantes diariamente en las escuelas es insuficiente, se aprende poco y eso repercute en acentuar las diferencias a nivel regional.

\section{Lograr una mayor igualdad entre las distintas zonas de un país}

Las zonas rurales y de asentamientos indígenas son las más desfavorecidas en cuanto a acceso y nivel educativo (las jornadas son de menos horas y los docentes son menos calificados, aunado a condiciones familiares y culturales que dificultan una educación de calidad).

Las preocupaciones incluyen no solo luchar contra las diferencias en cobertura o calidad educativa, sino que también se debe luchar contra las condiciones precarias de vida, pues existen altos índices de desnutrición, insalubridad y enfermedades. 
Walter (2000) sabiamente plantea que “(...) un buen desayuno para una alumna puede significar la diferencia entre una mañana de clases bien aprovechada o la pérdida de varias horas de tiempo para el maestro y para la niña" (p. 37).

Igualdad de oportunidades, que es algo positivo que se pretende alcanzar, no significa que todos se rijan por el mismo currículo, este puede variar según la región para darle sentido a la enseñanza.

\section{Lograr una mayor igualdad entre la educación pública y privada}

Generalmente las escuelas privadas están dotadas de una buena infraestructura y materiales adecuados. Por el contrario, las instituciones públicas tienen múltiples carencias que dificultan el aprendizaje. Muchas veces nos encontramos noticias que mencionan que los niños reciben clases en aulas improvisadas, en salones comunales de iglesias o en gimnasios y galerones, con pisos en mal estado, sin zonas verdes, sin divisiones adecuadas que generan ruidos, entre otras cosas.

Lo anterior trae como consecuencia que maestros y alumnos se desmotiven y estos últimos bajen su rendimiento académico, trayendo como consecuencia situaciones más graves como la repitencia y la deserción que, como menciona Walter (2000), son un lastre generador de grandes pérdidas económicas.

Por lo anterior, debería, a nivel estatal, existir una preocupación por equiparar lo más posible la educación pública con la privada, subsanando las deficiencias y carencias en cuanto a materiales e infraestructura para luego analizar las diferencias en los programas, tiempo lectivo efectivo y metodologías utilizadas.

\section{Brindar atención tomando en cuenta la diversidad}

Si bien es cierto que a estudiantes con problemas debe brindárseles atención inmediata y existen mecanismos para hacerlo, se considera también importante la atención a estudiantes talentosos, quienes muchas veces quedan desatendidos por priorizar los otros casos.

Es digno de mencionar que, en Costa Rica, se aprobó la ley \#8899 impulsada por la UNED llamada: Ley para la Promoción de la Alta Dotación, Talentos y Creatividad en el Sistema Educativo Costarricense, (Asamblea Legislativa de la República de Costa Rica, 2010), la cual en el artículo 1 plantea que :

La presente Ley tiene por objeto la promoción de la alta dotación, talentos y creatividad de los educandos con capacidades extraordinarias en el Sistema Educativo Costarricense. Estos estudiantes serán objeto de una atención temprana, individualizada, completa y oportuna por parte del Ministerio de Educación Pública. (ARTÍCULO 1.- Objeto, párr. 1)

Aquí se debe reiterar la importancia de docentes capacitados que atiendan a esta población estudiantil, pues el artículo 4 de dicha ley establece la necesidad de una flexibilización curricular $\mathrm{y}$ enuncia que: 
Los estudiantes con alta dotación, talentos y creatividad contarán con flexibilizaciones curriculares, esto de acuerdo con los procedimientos que el Estado establezca. Dicha flexibilización se les aplicará en el centro de estudio al que pertenece el educando o en centros que por sus condiciones puedan resultar más adecuados de acuerdo con las necesidades del estudiante (ARTÍCULO 4.- Flexibilización curricular, párr. 1).

La diversidad en el aula incluye diferentes ritmos y estilos de aprendizaje, pues todos y todas tienen derecho a una educación que se adapte a sus necesidades.

\section{Aprender a vivir juntos}

Este punto es crucial, si quiere que la globalización y las aceleradas transformaciones en la sociedad no borren el compromiso que se tiene con los otros, constituyéndose, como menciona Tedesco (2003), en un objetivo de aprendizaje y de política educativa para poder formar una sociedad más justa y solidaria. Autonomía y convivencia no son antónimos, pueden vincularse para formar un ser integral que, según Naranjo (2004), se forme en una educación orientada hacia la paz y hacia la unidad mundial.

\section{Pensar cambios a largo plazo. La educación del futuro}

Hay cambios que, como se planteó en el apartado anterior, se tornan urgentes, pero también debe existir una planificación que permita seguir implementándolos a mediano y largo plazo.

La educación en el futuro inmediato y a largo plazo debe ser una educación auténticamente humanizante (Hoyos et al., s. f.), con un proceso de evaluación continua que permita ir realizando las transformaciones emergentes.

Por lo anterior, se requiere de un currículo flexible y se debe como país estar anuentes a implementar los cambios que va demandando la nueva sociedad, de forma tal que la educación realmente vincule la escuela con estas necesidades de la sociedad y las empresas.

Esta educación demandará un docente que esté involucrado cognitiva y afectivamente con sus estudiantes, donde ambos aprenden en el proceso y el docente sea un profesional valorado y con un salario digno de su labor.

Esta educación debe pensarse, según Hoyos et al. (s.f.), “(...) para el desarrollo sostenible (...) [que lo cual implica instaurar] programas que sean localmente relevantes y culturalmente apropiados (...)” (p. 6), los cuales tomen en cuenta las condiciones ambientales, económicas y sociales de la localidad. Una educación también orientada a la transdisciplinariedad y el pensamiento complejo que permita enfrentar los cambios e incertidumbres del entorno, por tanto, que enseñe para la comprensión.

La imagen de una sociedad fragmentada, de un conocimiento parcelado y de saberes aislados no "encaja" dentro de esta nueva perspectiva.

Costa Rica ha realizado enormes esfuerzos por mejorar el sistema educativo, proclamando el derecho de todas las personas a una educación de calidad, integral y atractiva que "(...) supone, como mínimo, la universalización de la educación media y un acceso creciente a niveles superiores 
de educación, más sofisticados y diversos" (Consejo Superior de Educación, 2008, p. 6), donde la educación esté contextualizada y exista coherencia entre teoría y práctica, que haya la misma responsabilidad de todos y el estudiante sea un ente activo en este proceso. Como se puede observar, las pautas son claras, pero falta más acción.

Al respecto, Morin, Ciurana y Motta (2006) hablan de alcanzar una civilización planetaria, donde la educación tenga como misión el fortalecimiento de una sociedad-mundo e involucre a todos como protagonistas. Este sería el ideal...

\section{Conclusiones}

No se debe olvidar, como menciona Ruiz (2006), que existe una correlación entre escolaridad y desarrollo, y por ende entre ausencia de escolaridad y ausencia de oportunidades para las personas, lo cual repercute en la sociedad. En otras palabras, la educación es un factor importante para garantizar la movilidad social y equiparar desigualdades.

Gómez (2010) afirma que esta es determinante en cómo los sujetos comprenden el mundo e interactúan con él. O en palabras de Morin (1999): “(...) La educación es 'la fuerza del futuro', porque ella constituye uno de los instrumentos más poderosos para realizar el cambio (...)” (p. 7).

Por tanto, debemos tener claro que la educación es un aspecto crucial y no se puede seguir con los mismos patrones de hace 50 años; el mundo cambia y sus habitantes con él.

Por ende, se debe apostar por una educación que transforme integralmente al individuo y lo concientice de su responsabilidad para con él, con los otros y con el planeta.

Si queremos aumentar los niveles de promoción debemos resolver los múltiples problemas que hoy día viene arrastrando el sistema educativo en cuanto a reformar el currículo de acuerdo con los requerimientos del mundo tecnológico y globalizado en el que se vive actualmente, priorizar la capacitación y formación docente, ampliar la calidad y la cobertura escolar, garantizar al menos 12 años de enseñanza obligatoria y muchas otras que han sido mencionadas previamente.

Sería injusto decir que no se han realizado esfuerzos, pero aún seguimos siendo la región con mayores desigualdades educativas.

Pensar en la educación es algo que siempre debe estar presente en toda agenda gubernamental y en cada cabeza de los formadores, pues la educación no es un gasto, es una inversión.

\section{Referencias bibliográficas}

Arias, J. P. y EFE. (27 de octubre de 2010). Índice de prosperidad Legatum. País destaca entre los más prósperos de Latinoamérica. La Nación. Recuperado de http://www.nacion.com/2010-1027/Economia/NotasSecundarias/Economia2568779.aspx

Asamblea Legislativa de la República de Costa Rica. (28 de octubre 2010). Ley para la promoción de la alta dotación, talentos y creatividad en el sistema educativo costarricense (Ley $\mathrm{N}^{\circ}$ 8899). La Gaceta Diario Oficial \# 247 del 21 de diciembre del 2010. Recuperado de http://www. gaceta.go.cr/pub/2010/12/21/COMP_21_12_2010.html 
Consejo Superior de Educación. (2008). El centro educativo de calidad como eje de la educación costarricense. Recuperado de http://www.uned.ac.cr/educacion/documents/doc2011 yrivera/ un centro educativo de calidad.pdf

De Zubiría, J. (s. f). Los retos a la educación en el siglo XXI. Recuperado de http://www. institutomerani.edu.co/publicaciones/articulos/2009/Retos\%20a\%201a\%20educacion\%20 del\%20Siglo\%20XXI\%20De\%20Zubiria.pdf

Gómez, T. (2010). El nuevo paradigma de la complejidad y la educación: Una mirada histórica. Polis Revista de la Universidad Bolivariana, 9(25), 183-198.

Hoyos, C. A., Delgado, V. y Pére, D. L. (s. f). Tendencias mundiales en educación. Monografías. com Recuperado de http://www.uh.cu/static/documents/AL/Tendencias\%20mundiales\%20 educacin\%20mono.pdf

Morin, E. (1999). Los siete saberes necesarios para la educación del futuro. Francia: UNESCO. Recuperado de http://unesdoc.unesco.org/images/0011/001177/117740so.pdf

Morin, E., Ciurana, E. R. y Motta, R. D. (2006). Educar en la era planetaria. El pensamiento complejo como método de aprendizaje en el error y la incertidumbre humana. España: Gedisa.

Naranjo, C. (2004). Cambiar la educación para cambiar el mundo. Recuperado de http://www. claudionaranjo.net/pdf files/education/cambiar la educacion ch 5 spanish.pdf

PNUD (2010). Análisis de las tendencias de 40 años revela avances más rápidos en desarrollo en países pobres (Informe). Recuperado de http://hdr.undp.org/es/informes/mundial/idh2010/ noticias/title, 21577,es.html

Rivera, L. (2005). El papel del docente como gestor en el contexto actual. Revista de la Universidad Cristóbal Colón, 17-18, Recuperado de www.eumed.net/rev/rucc/17-18/

Ruiz, Á. (2006). Universalización de la educación secundaria y reforma educativa. San José, Costa Rica: EUCR. Recuperado de http://cimm.ucr.ac.cr/universalizacion/presentacion digital.html

Tedesco, J. C. (2003, octubre). Los pilares de la educación del futuro. Debats D‘Educació, 1-9. Recuperado de http://www.uoc.edu/dt/20367/20367.pdf

Walter, K. (2000). La educación en Centroamérica: Reflexiones en torno a sus problemas y su potencial. Recuperado de http://ca2020.fiu.edu/Themes/Knut Walter/Walter.pdf 